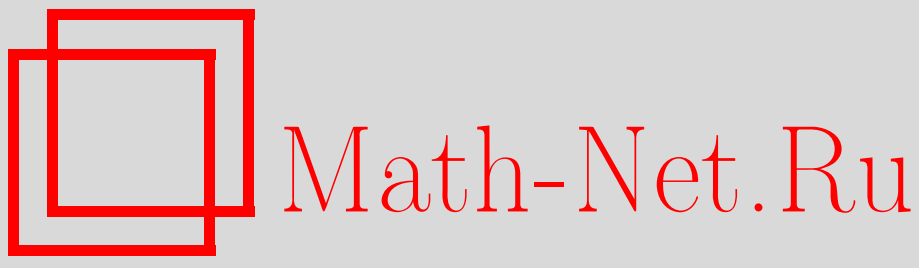

Р. Р. Гадыльшин, А. М. Ильин, Асимптотика собственных значений задачи Дирихле в области с узкой щелью, Матем. сб., 1998, том 189, номер 4, 25-48

DOI: https://doi.org/10.4213/sm305

Использование Общероссийского математического портала Math-Net.Ru подразумевает, что вы прочитали и согласны с пользовательским соглашением http://www . mathnet.ru/rus/agreement

Параметры загрузки:

IP: 54.84 .234 .179

26 апреля 2023 г., 15:01:43 
УДК 517.956

\author{
Р.Р. Гадыльшин, А.М. Ильин
}

\title{
Асимптотика собственных значений задачи Дирихле в области с узкой щелью
}

\begin{abstract}
Рассматривается задача Дирихле в двумерной области с узкой щелью, ширина которой является мальм параметром. Методом согласования асимптотических разложений строится полное асимптотическое разложение собственного значения возмущенной задачи, сходящегося к простому собственному значению предельной задачи. Показано, что регулярная теория возмущений может быть формально проведена естественным образом вплоть до величины $\varepsilon^{2}$, но полученный таким образом результат неверен. Только привлечение внутреннего асимптотического разложения позволяет получить правильный ответ.

Библиографйя: 14 названий.
\end{abstract}

\section{Введение}

Исследованию асимптотик собственных значений эллиптических краевых задач при различных типах сингулярных возмущений граничных условий посвящено достаточно много работ (см., например, [1]-[8] и содержащийся в этих работах обзор литературы). Ниже рассмотрена задача Дирихле в двумерной области с узкой шелью, именуемая в дальнейшем возмущенной задачей. Мальм параметром является ширина шели. Такая задача является бисингулярной - коэффициенты внешнего разложения имеют нарастающие особенности вблизи концов разреза, к которому стягивается щель. В настоящей работе методом согласования асимптотических разложений [9]-[12] строится полное асимптотическое разложение собственного значения возмушенной задачи, сходяшегося к простому собственному значению предельной задачи. Методика исследования имеет сходные черты с методикой статьи [9], где предельная задача считалась однозначно разрешимой, однако есть и существенные отличия. Вследствие того, что надо одновременно с асимптотикой решения (собственной функции) получать и асимптотику собственного значения, здесь затруднительно строить сначала внешнее асимптотическое разложение и затем "исправлять" его с помощью внутреннего. Более того, ниже (в $§ 3$ ) показывается, что регулярная теория возмушений может быть формально проведена естественньм образом вплоть до величины $\varepsilon^{2}$. Но выясняется, что полученный таким образом результат неверен. Только привлечение внутреннего асимптотического разложения позволяет получить правильный ответ.

Исследование можно провести для любого эллиптического оператора второго порядка, однако, чтобы сделать изложение менее громоздким, мы рассматриваем

Работа выполнена при финансовой поддержке Российского фонда фундаментальных исследований (гранты № 96-01-00501 и № 96-15-96241). 
лишь оператор Лапласа. Дополнительной причиной такого выбора является то, что для оператора Лапласа получаются явные нетривиальные формулы асимптотики собственного значения. В том случае, когда граница щели вблизи концов совпадает с параболами, собственное значение разлагается в асимптотический ряд по цельм степеням $\varepsilon$. В общем случае гладкой границы щели в асимптотике собственного значения присутствуют члены $\varepsilon^{k} \ln ^{j} \varepsilon$, где $j \leqslant[(k-2) / 6]$ (квадратные скобки означают целую часть), так что наибольший по величине логарифмический член - это $\varepsilon^{8} \ln \varepsilon$. С другой стороны, все коэффициенты при $\varepsilon^{6 m+2} \ln ^{m} \varepsilon$, вообще говоря, не равны нулю. Заметим, что асимптотика решения первой краевой задачи для уравнения Пуассона в рассматриваемой области не содержит степеней $\ln \varepsilon$ для любой формы щели [10], а в статье [9] показано, что для эллиптического оператора второго порядка асимптотика решения вне конщов шели содержит, вообще говоря, члены вида $\varepsilon^{k} \ln ^{j} \varepsilon$ при $j \leqslant[(k-2) / 4]$.

\section{§1. Постановка задачи}

Пусть $\Omega$ - ограниченная, односвязная область в $\mathbb{R}^{2}$, имеющая бесконечно дифференцируемую границу $\Gamma$, $\omega_{0}$ - интервал $(0,1)$ на оси $O x_{1}, \bar{\omega}_{0} \subset \Omega$, $\omega_{\varepsilon}=\left\{x: 0<x_{1}<1, \varepsilon g_{-}\left(x_{1}\right)<x_{2}<\varepsilon g_{+}\left(x_{1}\right)\right\}$, где $0<\varepsilon \ll 1, g_{ \pm} \in C^{\infty}\left(\omega_{0}\right)$,

$$
\lim _{t \rightarrow 0} g_{ \pm}(t)=\lim _{t \rightarrow 1} g_{ \pm}(t)=0
$$

Обозначим $\Omega_{\delta}=\Omega \backslash \bar{\omega}_{\delta}, \Gamma_{\delta}=\partial \Omega_{\delta}, \delta \geqslant 0$. В введенных обозначениях предельную и возмушенную задачи на собственные значения можно записать в единой форме:

$$
-\Delta \varphi_{\delta}=\lambda_{\delta} \varphi_{\delta}, \quad x \in \Omega_{\delta}, \quad \varphi_{\delta}=0, \quad x \in \Gamma_{\delta}
$$

где $\delta=0$ соответствует предельной задаче, а $\delta=\varepsilon>0$ - возмушенной.

Вблизи концов отрезка будем предполагать границу щели $\omega_{\varepsilon}$ гладкой. Это означает, что около точек $(0,0)$ и $(1,0)$ уравнение границы имеет вид: $x_{1}=G_{-}\left(x_{2}\right)$ и $x_{1}=G_{+}\left(x_{2}\right)$, соответственно, где $G_{ \pm} \in C^{\infty}$. Ясно, что

$$
G_{-}(0)=G_{-}^{\prime}(0)=G_{+}^{\prime}(0)=0, \quad G_{+}(0)=1, \quad \pm G_{ \pm}^{\prime \prime}(0) \leqslant 0 .
$$

Дополнительно предположим, что $G_{-}^{\prime \prime}(0) G_{+}^{\prime \prime}(0) \neq 0$. В этом случае функции $g_{ \pm}$ имеют на концах интервала $\omega_{0}$ следующие асимптотики:

$$
\begin{aligned}
& g_{ \pm}\left(x_{1}\right)=\sum_{j=1}^{\infty}( \pm 1)^{j} g_{j}^{-} x_{1}^{j / 2}, \quad x_{1} \rightarrow 0 \\
& g_{ \pm}\left(x_{1}\right)=\sum_{j=1}^{\infty}( \pm 1)^{j} g_{j}^{+}\left(1-x_{1}\right)^{j / 2}, \quad x_{1} \rightarrow 1,
\end{aligned}
$$

где $g_{1}^{ \pm}=\sqrt{\mp 2 / G_{ \pm}^{\prime \prime}(0)}$, и следовательно, $g_{1}^{ \pm}>0$. 


\section{§ 2. Равномерные оценки решений возмущенной задачи}

Решения как предельной, так и возмушенной краевых задач удобно рассматривать в классе обобшенных решений из пространств Соболева (см., например, [13]). Для области $Q$ обозначим через $\stackrel{\circ}{W} \underset{2}{1}(Q)$ - замыкание функций из $C_{0}^{\infty}(Q)$ по норме $W_{2}^{1}(Q)$, а через $(\cdot, \cdot)_{Q}$ - скалярное произведение в $L_{2}(Q)$. Под решением краевой задачи

$$
-\Delta u_{\delta}=\lambda u_{\delta}+f_{\delta}, \quad x \in \Omega_{\delta}, \quad u_{\delta}=0, \quad x \in \Gamma_{\delta},
$$

где $f_{\delta} \in L_{2}\left(\Omega_{\delta}\right)$, понимается элемент $u_{\delta} \in \stackrel{\circ}{W} \underset{2}{1}\left(\Omega_{\delta}\right)$, удовлетворяющий интегральному тождеству

$$
\left(\nabla u_{\delta}, \nabla v\right)_{\Omega_{\delta}}=\left(\lambda u_{\delta}+f_{\delta}, v\right)_{\Omega_{\delta}}
$$

при любом $v \in \stackrel{\circ}{W} \underset{2}{2}\left(\Omega_{\delta}\right)$. Всюду далее функции из $L_{2}\left(\Omega_{\varepsilon}\right)$ и $\stackrel{\circ}{W} \underset{2}{1}\left(\Omega_{\varepsilon}\right)$ считаются продолженными нулем в $\omega_{\varepsilon}$. Именно в таком контексте понимаются очевидные включения $\stackrel{\circ}{W} \frac{1}{2}\left(\Omega_{\mu}\right) \subset \stackrel{\circ}{W} \frac{1}{2}\left(\Omega_{\nu}\right) \subset \stackrel{\circ}{W} \frac{1}{2}(\Omega)$ и $L_{2}\left(\Omega_{\mu}\right) \subset L_{2}\left(\Omega_{\nu}\right)$ для любых $0 \leqslant \nu<\mu$. Ясно также, что $L_{2}\left(\Omega_{0}\right) \subset L_{2}(\Omega)$. Обозначим через $\|\cdot\|_{Q}$ и $\|\cdot\|_{m, Q}$ нормы в $L_{2}(Q)$ и $W_{2}^{m}(Q)$, соответственно.

Пусть $\lambda_{\delta}^{(n)}$ - упорядоченные по возрастанию собственные значения (1.1) с учетом их кратности, а $\varphi_{\delta}^{(n)}-$ соответствующие ортонормированные в $L_{2}(\Omega)$ собственные функции. Из вариационного принципа и теорем вложения вытекает

ЛЕмма 2.1. Для любых $0 \leqslant \nu<\mu$ имеют место неравенство $0<\lambda_{\nu}^{(n)}<\lambda_{\mu}^{(n)}$ и сходимость $\lambda_{\varepsilon}^{(n)} \rightarrow \lambda_{0}^{(n)}$ при $\varepsilon \rightarrow 0$. Если $\lambda_{0}^{(n)}-$ простое собственное значение,

Всюду далее будем обозначать через $\lambda_{0}$ простое собственное значение предельной задачи, а через $\lambda_{\varepsilon}$ - простое собственное значение возмушенной задачи, сходяшееся к $\lambda_{0}$. Аналогично, через $\varphi_{0}$ и $\varphi_{\varepsilon}$ будем обозначать соответствуюшие нормированные в $L_{2}(\Omega)$ собственные функции.

Лемма 2.2. Пусть $u_{\varepsilon}-$ решение (2.1) при $\lambda=\lambda_{\varepsilon} u\left(u_{\varepsilon}, \varphi_{\varepsilon}\right)_{\Omega}=0$. Тогда справедлива оченка

$$
\left\|u_{\varepsilon}\right\|_{1, \Omega} \leqslant C\left\|f_{\varepsilon}\right\|_{\Omega}
$$

где постоянная $C$ не зависит от $\varepsilon$.

ДокАЗАТЕЛЬСтво. Легко видеть, что из (2.2) вытекает равномерная априорная оценка

$$
\left\|u_{\varepsilon}\right\|_{1, \Omega} \leqslant C\left(\left\|u_{\varepsilon}\right\|_{\Omega}+\left\|f_{\varepsilon}\right\|_{\Omega}\right) .
$$

Дальнейшее доказательство будем вести от противного. Допустим, сушествует последовательность $\varepsilon_{n} \rightarrow 0$ при $n \rightarrow \infty$ такая, что при $\varepsilon_{n}=\varepsilon$ имеет место оценка

$$
\left\|u_{\varepsilon}\right\|_{1, \Omega} \geqslant n\left\|f_{\varepsilon}\right\|_{\Omega}
$$


для некоторых $f_{\varepsilon}$ (для $0<\varepsilon_{0}<\varepsilon \leqslant \delta_{0}$ равномерная оценка $(2.3)$ вытекает из известных априорных оценок решений эллиптических уравнений в области с гладкой границей). Не ограничивая общности, будем считать, что $\left\|u_{\varepsilon}\right\|_{\Omega}=1$. Из (2.4) и (2.5) следует, что

$$
\left\|u_{\varepsilon}\right\|_{1, \Omega} \leqslant T
$$

где $T$ - некоторая фиксированная постоянная. Заметим, что из (2.5) и (2.6) следует сходимость $f_{\varepsilon}$ к нулю в норме $L_{2}(\Omega)$. Из слабой компактности ограниченного множества в $W_{2}^{1}(\Omega)$ и его же компактности в $L_{2}(\Omega)$ вытекает, что существует подпоследовательность $\varepsilon_{n^{\prime}} \rightarrow 0$, на которой функции $u_{\varepsilon}$ сходятся к нетривиальному решению $u_{0} \neq 0$ предельной задачи для $f_{0}=0$ и $\lambda=\lambda_{0}$. Причем, в силу ортогональности $\left(u_{\varepsilon}, \varphi_{\varepsilon}\right)_{\Omega}=0$ и леммы 2.1 имеет место равенство $\left(u_{0}, \varphi_{0}\right)_{\Omega}=0$. Полученное противоречие доказывает оценку (2.3). Лемма доказана.

ЛЕмма 2.3. При $\lambda$, близких $\kappa \lambda_{0}$, для решения задачи (2.1) справедливы равномерние оценки

$$
\begin{gathered}
\left|\lambda_{\varepsilon}-\lambda\right|\left|\left(u_{\varepsilon}, \varphi_{\varepsilon}\right)_{\Omega}\right| \leqslant\left\|f_{\varepsilon}\right\|_{\Omega}, \\
\left\|u_{\varepsilon}-\left(\varphi_{\varepsilon}, u_{\varepsilon}\right)_{\Omega} \varphi_{\varepsilon}\right\|_{1, \Omega} \leqslant C\left\|f_{\varepsilon}\right\|_{\Omega} .
\end{gathered}
$$

ДокАЗАТЕЛЬСТво. Подставляя $v=\varphi_{\varepsilon}$ в $(2.2)$ и учитывая равенство

$$
\left(\nabla \varphi_{\varepsilon}, \nabla u_{\varepsilon}\right)_{\Omega}=\lambda_{\varepsilon}\left(\varphi_{\varepsilon}, u_{\varepsilon}\right)_{\Omega}
$$

получаем оценку (2.7). В свою очередь, применяя утверждение леммы 2.2 к функции $u_{\varepsilon}-\left(\varphi_{\varepsilon}, u_{\varepsilon}\right)_{\Omega} \varphi_{\varepsilon}$ и используя оценку $(2.7)$, получаем оценку (2.8). Лемма доказана.

\section{§3. Регулярная теория возмущений}

Из леммы 2.1 следует, что собственная функция $\varphi_{\varepsilon}$ сходится к $\varphi_{0}$. Поэтому главный член асимптотики $\varphi_{\varepsilon}$ равен $\varphi_{0}$. Представляется естественным асимптотики $\varphi_{\varepsilon}$ и $\lambda_{\varepsilon}$ искать в следуюшем виде:

$$
\begin{aligned}
\varphi_{\varepsilon}(x) & =\varphi_{0}(x)+\varepsilon \varphi_{1}(x)+\varepsilon^{2} \varphi_{2}(x)+\cdots, \\
\lambda_{\varepsilon} & =\lambda_{0}+\varepsilon \lambda_{1}+\varepsilon^{2} \lambda_{2}+\cdots .
\end{aligned}
$$

Подставляя (3.1) и (3.2) в (1.1), получаем краевые задачи для коэффициентов $\varphi_{1}$ и $\varphi_{2}$ :

$$
\begin{gathered}
-\left(\Delta+\lambda_{0}\right) \varphi_{1}=\lambda_{1} \varphi_{0}, \quad x \in \Omega_{0}, \\
\varphi_{1}=0, \quad x \in \partial \Omega, \\
\varphi_{1}=-g_{ \pm}\left(x_{1}\right) \frac{\partial \varphi_{0}}{\partial x_{2}}, \quad x_{1} \in \omega_{0}, \quad x_{2}= \pm 0, \\
-\left(\Delta+\lambda_{0}\right) \varphi_{2}=\lambda_{1} \varphi_{1}+\lambda_{2} \varphi_{0}, \quad x \in \Omega_{0}, \\
\varphi_{2}=0, \quad x \in \partial \Omega, \\
\varphi_{2}=-g_{ \pm}\left(x_{1}\right) \frac{\partial \varphi_{1}}{\partial x_{2}}-\frac{1}{2} g_{ \pm}^{2}\left(x_{1}\right) \frac{\partial^{2} \varphi_{0}}{\partial x_{2}^{2}}, \quad x_{1} \in \omega_{0}, \quad x_{2}= \pm 0 .
\end{gathered}
$$


Пусть $r, \theta$ - полярные координаты. Обозначим через $\Psi_{k}(\theta)$ линейную комбинацию $\sin (j \theta / 2)$ и $\cos (j \theta / 2)$, где $0 \leqslant j \leqslant k$ и $j \equiv k(\bmod 4)$. Из [9], [14] вытекает следуюший упрощенньй аналог лемм 1 и 2 работы [9].

ЛЕмма 3.1. Пусть функции $f \in W_{2}^{1}\left(\Omega_{0}\right) \cap C^{\infty}\left(\Omega_{0}\right)$ и $h_{ \pm} \in C^{\infty}\left(\omega_{0}\right)$ имеют при $r \rightarrow 0$ и при $x_{1} \rightarrow 0$ асимптотики

$$
\begin{aligned}
f(x) & =\sum_{k=0}^{\infty} r^{k / 2} \Psi_{k}(\theta), \\
h_{ \pm}\left(x_{1}\right) & =\sum_{k=0}^{\infty} b_{k}\left( \pm x_{1}\right)^{k / 2}
\end{aligned}
$$

и аналогичные разложения при $\left(1-x_{1}\right)^{2}+x_{2}^{2} \rightarrow 0 u x_{1} \rightarrow 1$. Далее, пусть $u \in W_{2}^{1}\left(\Omega_{0}\right)$ - решение краевой задачи

$$
\begin{gathered}
-(\Delta+\lambda) u=f, \quad x \in \Omega_{0}, \\
u=0, \quad x \in \partial \Omega, \\
u=h_{ \pm}, \quad x_{1} \in \omega_{0}, \quad x_{2}= \pm 0 .
\end{gathered}
$$

Тогда и $\in C^{\infty}\left(\Omega_{0}\right)$ также имеет асимптотики вида (3.5) в окрестности концов разреза $\omega_{0}$, причем, $\Psi_{0}=b_{0}$.

Если $f=0$ и $h_{ \pm}=0$, то суммирование в (3.5) начинается с $k=1 u$ $\Psi_{1}(\theta)=\alpha \sin (\theta / 2)$.

Если $\lambda$ не является собственным значением предельной задачи, то существует решение $u \in W_{2}^{1}\left(\Omega_{0}\right)$, удовлетворяющее всем выше приведенным утверждениям леммы.

Здесь и всюду далее предполагается, что асимптотические ряды по переменным $x_{1}$ и $x_{2}$ бесконечно дифференцируемы.

СлЕДСТВИЕ. Собственная функиия $\varphi_{0}$ имеет в окрестности концов разреза $\omega_{0}$ разложсения вида (3.5), әде суммирование начинается с $k=1$.

ЛЕмма 3.2. Существует функция $\varphi_{1} \in W_{2}^{1}\left(\Omega_{0}\right) \cap C^{\infty}\left(\Omega_{0}\right)$, имеющая асимптотики (3.5) и являющаяся решением краевой задачи (3.3) при

$$
\lambda_{1}=\int_{0}^{1}\left(g_{+}\left(x_{1}\right)\left(\frac{\partial}{\partial x_{2}} \varphi_{0}\left(x_{1},+0\right)\right)^{2}-g_{-}\left(x_{1}\right)\left(\frac{\partial}{\partial x_{2}} \varphi_{0}\left(x_{1},-0\right)\right)^{2}\right) d x_{1} .
$$

ДокАЗАТЕЛЬСТво. Из следствия леммы 3.1 вытекает, что правые части в уравнении и в граничном условии на $\omega_{0}$ в (3.3) удовлетворяют условию леммы 3.1. Следовательно, для любого $\lambda$, не являюшегося собственным значением, сушествует решение краевой задачи

$$
\begin{gathered}
-(\Delta+\lambda) u=0, \quad x \in \Omega_{0}, \\
u=0, \quad x \in \partial \Omega \\
u=-g_{ \pm} \frac{\partial \varphi_{0}}{\partial x_{2}}, \quad x_{1} \in \omega_{0}, \quad x_{2}= \pm 0
\end{gathered}
$$


удовлетворяюшее всем утверждением леммы 3.1. Представляя решение задачи (3.3) в виде $\varphi_{1}=u+w$, для функции $w$ получаем следующую краевую задачу:

$$
\begin{gathered}
-\left(\Delta+\lambda_{0}\right) w=\left(\lambda_{0}-\lambda\right) u+\lambda_{1} \varphi_{0}, \quad x \in \Omega_{0}, \\
w=0, \quad x \in \partial \Omega_{0} .
\end{gathered}
$$

Так как правая часть уравнения в (3.7) принадлежит $L_{2}(\Omega)$, то необходимым и достаточным условием разрешимости (3.7) является ортогональность в $L_{2}(\Omega)$ правой части и $\varphi_{0}$, которая достигается подходящим выбором постоянной $\lambda_{1}$. По построению функция $\varphi_{1}$ удовлетворяет всем условиям леммы 3.1. Соотношение (3.6) легко получается интегрированием по частям левой части тождества

$$
-\left(\left(\Delta+\lambda_{0}\right) \varphi_{1}, \varphi_{0}\right)_{\Omega_{0}}=\lambda_{1}\left(\varphi_{0}, \varphi_{0}\right)_{\Omega}=\lambda_{1}
$$

Лемма доказана.

Так как функция $\varphi_{1}$ определена с точностью до слагаемого $\alpha \varphi_{0}$, то для определенности выберем постоянную $\alpha$ из условия ортогональности: $\left(\varphi_{1}, \varphi_{0}\right)_{\Omega}=0$.

ЗАмЕчАниЕ 3.1. Ниже, из построения (и обоснования) полных асимптотических разложений собственного значения и собственной функции будет следовать, что полученные вьшше коэффициенты $\varphi_{1}$ и $\lambda_{1}$-верные. С формальной точки зрения, используя регулярную теорию возмущений, можно определить и коэффициенты $\varphi_{2}$ и $\lambda_{2}$. Действительно, в силу уравнения второе слагаемое в граничном условии для $\varphi_{2}$ равно нулю, и следовательно, правые части в (3.4) удовлетворяют условиям леммы 3.1. Повторяя доказательство леммы 3.2, получаем сушествование функции $\varphi_{2}=\widetilde{\varphi} \in W_{2}^{1}\left(\Omega_{0}\right) \cap C^{\infty}\left(\Omega_{0}\right)$, имеющей асимптотику $(3.5)$ и являюшейся решением краевой задачи (3.4) при $\lambda_{2}=\widetilde{\lambda}$, где

$$
\begin{aligned}
\tilde{\lambda}=\int_{0}^{1}\left(g _ { + } ( x _ { 1 } ) \frac { \partial } { \partial x _ { 2 } } \varphi _ { 1 } \left(x_{1},\right.\right. & +0) \frac{\partial}{\partial x_{2}} \varphi_{0}\left(x_{1},+0\right) \\
& \left.-g_{-}\left(x_{1}\right) \frac{\partial}{\partial x_{2}} \varphi_{1}\left(x_{1},-0\right) \frac{\partial}{\partial x_{2}} \varphi_{0}\left(x_{1},-0\right)\right) d x_{1} .
\end{aligned}
$$

Однако, как это будет показано ниже, приведенные значения $\lambda_{2}=\widetilde{\lambda}$ и $\varphi_{2}=\widetilde{\varphi}$ не являются верными.

\section{§4. Построение вторых членов асимптотик методом согласования асимптотических разложений}

Для правильного определения $\lambda_{2}$ надо использовать внутреннее асимптотическое разложение в окрестности концов отрезка. Вид этого разложения определяется (в соответствии с методом согласования) из уже построенных $\varphi_{0}$ и $\varphi_{1}$. В силу лемм 3.1 и 3.2 и формул (3.1) и (1.2) в начале координат справедливы асимптотики

$$
\varphi_{0}(x)=d_{-} r^{1 / 2} \sin \frac{\theta}{2}+O(r), \quad \varphi_{1}(x)=-\frac{1}{2} d_{-} g_{1}^{-}+O\left(r^{1 / 2}\right)
$$


Такая же асимптотика (в соответствующей полярной системе координат и с заменой постоянных $d_{-}$и $g_{1}^{-}$на $d_{+}$и $\left.g_{1}^{+}\right)$имеет место и в окрестности точки $(1,0)$.

В окрестности концов щели асимптотику решения будем искать в виде рядов по степеням $\varepsilon$, коэффициентами которых являются функции, зависящие от растянутых (внутренних) переменных $\xi_{j}=x_{j}\left(g_{1}^{-}\right)^{-2} \varepsilon^{-2}, j=1,2$, в окрестности левого конца и $\xi_{1}=\left(1-x_{1}\right)\left(g_{1}^{+}\right)^{-2} \varepsilon^{-2}, \xi_{2}=x_{2}\left(g_{1}^{+}\right)^{-2} \varepsilon^{-2}$ в окрестности правого конца. Переписьвая асимптотики $\varphi_{j}$ на концах щели во внутренних переменных, получаем, что

$$
\varphi_{0}(x)+\varepsilon \varphi_{1}(x)=\varepsilon d_{ \pm} g_{1}^{ \pm}\left(\rho^{1 / 2} \sin \frac{\theta}{2}-\frac{1}{2}\right)+O\left(\varepsilon^{2}\right),
$$

где $(\rho, \theta)$ - полярные координаты на плоскости $\xi=\left(\xi_{1}, \xi_{2}\right)$.

Равенство (4.1) приводит к мысли, что в окрестности концов щели собственная функция должна во внутренних переменных иметь следующий вид:

$$
\varphi_{\varepsilon}(x)=\varepsilon v_{1}^{ \pm}(\xi)+O\left(\varepsilon^{2}\right) .
$$

Краевые задачи для $v_{1}^{ \pm}$получаются стандартньм образом (см. [9], [10]). Подставляем (4.2) и (3.2) в (1.1) и переходим к внутренним переменным в уравнении и в граничных условиях, учитывая, что в окрестности левого конца уравнение щели имеет асимптотику

$$
\xi_{1}=\xi_{2}^{2}(1+o(1)), \quad \varepsilon \xi_{2} \rightarrow 0
$$

и аналогичный вид в окрестности другого конца. Оставляя члены при наименьшей степени $\varepsilon$, получаем следующие краевые задачи:

$$
\Delta v_{1}^{ \pm}=0, \quad \xi \in \Pi, \quad v_{1}^{ \pm}=0, \quad \xi \in \partial \Pi,
$$

где $\Pi=\left\{\xi: \xi_{1}<\xi_{2}^{2}\right\}$.

В силу (4.1) (и идеологии метода согласования асимптотических разложений) решения (4.3) должны иметь асимптотики

$$
v_{1}^{ \pm}(\xi)=d_{ \pm} g_{1}^{ \pm}\left(\rho^{1 / 2} \sin \frac{\theta}{2}-\frac{1}{2}\right)+o(1), \quad \rho \rightarrow \infty .
$$

Нетрудно выписать явный вид таких функций. Для этого введем комплексную переменную $y=\xi_{1}+\mathrm{i} \xi_{2}$ (здесь и далее $\mathrm{i}$ - мнимая единица). Легко видеть, что имеет место равенство

$$
\left.\left(y-\frac{1}{4}\right)^{1 / 2}\right|_{\xi_{1}=\xi_{2}^{2}}=\xi_{2}+\frac{\mathrm{i}}{2}
$$

(разрез взят вдоль луча $\left(\frac{1}{4}, \infty\right)$ вешественной оси). Следовательно, функции

$$
v_{1}^{ \pm}(\xi)=d_{ \pm} g_{1}^{ \pm}\left(\operatorname{Im}\left(y-\frac{1}{4}\right)^{1 / 2}-\frac{1}{2}\right)
$$

являются решениями задачи (4.3), имеющими асимптотики (4.4). Более того, из (4.6) следует, что

$$
v_{1}^{ \pm}(\xi)=d_{ \pm} g_{1}^{ \pm}\left(\rho^{1 / 2} \sin \frac{\theta}{2}-\frac{1}{2}+\frac{1}{8} \rho^{-1 / 2} \sin \frac{\theta}{2}\right)+O\left(\rho^{-3 / 2}\right), \quad \rho \rightarrow \infty .
$$


Переписывая теперь асимптотики $\varepsilon v_{1}^{ \pm}$на бесконечности во внешних переменных $x$, получаем главные члены асимптотик на концах щели для коэффициента $\varphi_{2}$ ряда (3.1):

$$
\varphi_{2}(x)=\frac{1}{8} d_{-}\left(g_{1}^{-}\right)^{2} r^{-1 / 2} \sin \frac{\theta}{2}+O(1)
$$

- на левом конце и аналогичньй вид на правом конце.

ЗАмЕчАНИЕ 4.1. Из асимптотики (4.8) следует, что $\varphi_{2}$ не может принадлежать (в случае достаточно общего положения $\left.\left|d_{+}\right|+\left|d_{-}\right| \neq 0\right)$ классу $W_{2}^{1}\left(\Omega_{0}\right)$. Именно по этой причине формально непротиворечивые регулярные построения вторых членов асимптотик, указанные в замечании 3.1 , приводят к неверным значениям искомых величин.

Перейдем к построению правильных значений для $\varphi_{2}$ и $\lambda_{2}$ в разложениях $(3.1)$ и (3.2). Для этого надо изменить $\widetilde{\varphi}$ и $\widetilde{\lambda}$, построенные в замечании 3.1 , учитывая асимптотику (4.8). Другими словами, надо к функции $\widetilde{\varphi}(x)$ добавить сингулярньй член const $r^{-1 / 2} \sin (\theta / 2)$ вблизи левого конща щели и аналогичньй член вблизи правого конца. Однако этим нельзя ограничиться. Для того чтобы функция $\varphi_{2}$ продолжала оставаться решением задачи (3.4), надо добавить дополнительное слагаемое из класса $W_{2}^{1}\left(\Omega_{0}\right)$.

Обозначим через $\left(r_{ \pm}, \theta_{ \pm}\right)$соответствующие полярные координаты в окрестности концов разреза $\omega_{0}$, а через $\chi(t)$ - бесконечно дифференцируемую срезающую функцию, тождественно равную единице при $t<c$ и нулю при $t>2 c$, где $c<\frac{1}{2}-$ достаточно малое число такое, что замкнутые круги радиуса $2 c$ с центрами в $(0,0)$ и $(1,0)$ лежат в $\Omega$.

Лемма 4.1. Существуют функиии

$$
\psi_{ \pm}(x)=\chi\left(r_{ \pm}\right) r_{ \pm}^{-1 / 2} \sin \frac{\theta_{ \pm}}{2}+\widetilde{\psi}_{ \pm}(x)
$$

әде $\widetilde{\psi}_{ \pm} \in W_{2}^{1}\left(\Omega_{0}\right)$, являющиеся решением краевой задачи

$$
-\left(\Delta+\lambda_{0}\right) \psi_{ \pm}=\lambda_{ \pm} \varphi_{0}, \quad x \in \Omega_{0}, \quad \psi_{ \pm}=0, \quad x \in \partial \Omega_{0},
$$

npu

$$
\lambda_{ \pm}=\pi d_{ \pm}
$$

ДокАЗАТЕльство. Будем искать $\widetilde{\psi}_{ \pm}$в следующем виде:

$$
\widetilde{\psi}_{ \pm}(x)=-\frac{\lambda_{0}}{2} \chi\left(r_{ \pm}\right) r_{ \pm}^{3 / 2} \sin \frac{\theta_{ \pm}}{2}+\widehat{\psi}_{ \pm}(x)
$$

Подставляя выражение $(4.9)$ в $(4.10)$, приходим к следующей задаче для $\widehat{\psi}_{ \pm}$:

$$
-\left(\Delta+\lambda_{0}\right) \widehat{\psi}_{ \pm}=\lambda_{ \pm} \varphi_{0}+f_{ \pm}, \quad x \in \Omega_{0}, \quad \widehat{\psi}_{ \pm}=0, \quad x \in \partial \Omega_{0}
$$

где $f_{ \pm} \in W_{2}^{1}(\Omega)$ имеют асимптотики (3.5). Достаточньм (и необходимым) условием разрешимости $(4.12)$ в $W_{2}^{1}\left(\Omega_{0}\right)$ является равенство $\lambda_{ \pm}=-\left(f_{ \pm}, \varphi_{0}\right)_{\Omega}$. 
Осталось получить соотношения (4.11). Обозначим $B^{ \pm}(t)=\Omega_{0} \backslash S_{ \pm}(t)$, где $S_{ \pm}(t)$ - круг радиуса $t$ с центром в соответствуюшем конще $\omega_{0}$. Так как функции $\widetilde{\psi}_{ \pm}$удовлетворяют утверждениям леммы 3.1 , то интегрируя по частям левые части тождеств

$$
-\left(\left(\Delta+\lambda_{0}\right) \psi_{ \pm}, \varphi_{0}\right)_{B^{ \pm}(t)}=\lambda_{ \pm}\left(\varphi_{0}, \varphi_{0}\right)_{B^{ \pm}(t)},
$$

учитывая (4.9) и асимптотику $\varphi_{0}(x)$ и переходя к пределу при $t \rightarrow 0$, получаем соотношения (4.11). Лемма доказана.

В силу леммы 4.1 и построений, приведенных в замечании 3.1 функция

$$
\varphi_{2}(x)=\frac{1}{8}\left(d_{+}\left(g_{1}^{+}\right)^{2} \psi_{+}(x)+d_{-}\left(g_{1}^{-}\right)^{2} \psi_{-}(x)\right)+\widetilde{\varphi}(x)
$$

является решением краевой задачи (3.4) при

$$
\lambda_{2}=\frac{\pi}{8}\left(\left(d_{+} g_{1}^{+}\right)^{2}+\left(d_{-} g_{1}^{-}\right)^{2}\right)+\widetilde{\lambda}
$$

где постоянная $\widetilde{\lambda}$ определяется равенством (3.8), и имеет на концах разреза требуемые асимптотики (4.8).

Таким образом, коэффициенты $\varphi_{2}$ и $\lambda_{2}$ рядов (3.1) и (3.2), а также главные члены $v_{1}^{ \pm}$внутренних разложений построены.

\section{§5. Внутреннее разложение в случае параболических концов щели}

Всюду в этом разделе предполагается, что граница щели в окрестности концов разреза имеет вид парабол. Будем искать асимптотику собственного значения и внутренние разложения в более развернутых, чем в (3.2) и в (4.2), видах:

$$
\begin{gathered}
\lambda_{\varepsilon}=\sum_{i=0}^{\infty} \varepsilon^{i} \lambda_{i}, \\
\varphi_{\varepsilon}(x)=\sum_{i=1}^{\infty} \varepsilon^{i} v_{i}^{ \pm}(\xi) .
\end{gathered}
$$

Так как приводимые ниже рассуждения для коэффициентов внутренних разложений - одни и те же в окрестности обоих концов щели, то, во-первых, ограничимся рассмотрением разложения на левом конце, а, во-вторых, чтобы не загромождать текст индексами “土”, будем их, по-возможности, опускать.

При наложенных на границу $\partial \omega_{\varepsilon}$ ограничениях ее уравнение в окрестности концов щели, записанное во внутренних переменных, имеет вид:

$$
\xi_{1}=\xi_{2}^{2}
$$


Подставляя (5.1)-(5.3) в (1.1), переходя к внутренним переменным и выписывая равенства при одинаковых степенях $\varepsilon$, получаем следуюшую рекуррентную систему краевых задач:

$$
\begin{gathered}
-\Delta v_{n}=\sum_{k=0}^{n-5} \lambda_{i} v_{n-k-4}, \quad \xi \in \Pi, \\
v_{n}=0, \quad \xi \in \partial \Pi .
\end{gathered}
$$

Пусть $z=\xi_{1}-\frac{1}{4}+\mathrm{i} \xi_{2}$.

Лемма 5.1. Для любых $k$ и $m$, отличных от -1 , уравнение $\Delta v=\bar{z}^{k} z^{m}$ имеет решение вида $v=c \bar{z}^{k+1} z^{m+1}$.

ЛЕмма 5.2. Для любого челого $k \geqslant 0$ краевая задача

$$
\Delta v=0, \quad \xi_{1}<\xi_{2}^{2}, \quad v=\xi_{2}^{k}, \quad \xi_{1}=\xi_{2}^{2},
$$

имеет решение вида

$$
v(\xi)=\sum_{j=0}^{[k / 2]} \alpha_{j} \operatorname{Re} z^{k / 2-j} .
$$

Справедливость этих двух лемм очевидным образом следует из равенства (4.5) и представления $\Delta=4 \frac{\partial^{2}}{\partial z \partial \bar{z}}$.

СлЕДСТвиЕ. Существуют решения краевъх задач

$$
\Delta X_{m}=0, \quad \xi \in \Pi, \quad X_{m}=0, \quad \xi \in \partial \Pi,
$$

которые могут бъть представлень в виде:

$$
X_{m}(\xi)=\operatorname{Im} z^{m / 2}+\sum_{j=0}^{[(m-1) / 2]} \alpha_{j} \operatorname{Re} z^{(m-1) / 2-j} .
$$

Отметим, что главный член асимптотики функции $X_{m}(\xi)$ на бесконечности имеет вид: $\rho^{m / 2} \sin (m \theta / 2)$.

В свою очередь, из утверждений лемм 5.1 и 5.2 вытекает

ЛЕмма 5.3. Пусть $1 \leqslant n<\infty, 1 \leqslant i \leqslant n, a\left\{a_{i}^{(n)}\right\}-$ произвольная последовательность действительных чисел. Тогда система краевых задач (5.4), (5.5) имеет систему решений, которая может бъть представлена в виде

$$
\begin{gathered}
v_{n}(\xi)=\sum_{i=1}^{n} a_{i}^{(n)} X_{i}(\xi)+\widetilde{v}_{n}(\xi) \\
\widetilde{v}_{n}(\xi)=\sum_{k=1}^{[(n-1) / 4]} \sum_{j=1}^{n-4 k}|z|^{2 k}\left(\alpha_{n, k, j} \operatorname{Im} z^{j / 2}+\beta_{n, k, j} \operatorname{Re} z^{(j-1) / 2}\right) \\
+\sum_{j=1}^{n} \beta_{n, 0, j} \operatorname{Re} z^{(j-1) / 2}
\end{gathered}
$$

где постоянные $\alpha_{n, k, j}$ и $\beta_{n, k, j}$ не зависят от $a_{s}^{(m)}$ nри $m \geqslant n$. 
ДоКАЗАТЕЛЬСТВо проводится по индукции. Для $n \leqslant 4$ уравнения (5.4) однородные, и согласно следствию леммы 5.2 существуют решения вида (5.6) с $\widetilde{v}_{n}(\xi) \equiv 0$. Для $n \geqslant 5$ уравнения (5.4) неоднородные, и для построения их решений следует учесть, что

$$
\Delta\left(|z|^{2 k} \operatorname{Im} z^{j / 2}\right)=4 \operatorname{Im} \frac{\partial^{2}}{\partial \bar{z} \partial z}\left(\bar{z}^{k} z^{k+j / 2}\right)=2 k(2 k+j)|z|^{2 k-2} \operatorname{Im} z^{j / 2}
$$

и аналогичное равенство справедливо для $|z|^{2 k} \operatorname{Re} z^{j / 2}$. Поэтому легко построить решение неоднородного уравнения (5.4) подобного вида. Для ликвидации возник-

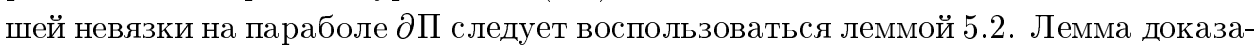
на.

\section{§6. Внешнее разложение в случае параболических концов щели}

Асимптотику собственной функции вне конщов шели (внешнее разложение) будем искать в виде ряда

$$
\varphi_{\varepsilon}(x)=\sum_{j=0}^{\infty} \varepsilon^{j} \varphi_{j} .
$$

Заметим, что асимптотическое разложение (6.1) (так же, как и асимптотическое разложение (5.2) из предыдущего параграфа) соответствует собственной функции $\varphi_{\varepsilon}$ c "нестрогой" нормировкой $\left\|\varphi_{\varepsilon}\right\|_{\Omega}=1+o(1)$ при $\varepsilon \rightarrow 0$.

Краевые задачи для коэффициентов рядов (5.1) получаются стандартной подстановкой рядов (5.1) и (6.1) в (1.1) с последующим вьписыванием равенств при одинаковых степенях $\varepsilon$ и формальньм переходом к пределу при $\varepsilon \rightarrow 0$. В результате этой процедуры получаем следующую рекуррентную систему краевых задач:

$$
\begin{gathered}
-\Delta \varphi_{n}=\sum_{j=0}^{n} \lambda_{j} \varphi_{n-j}, \quad x \in \Omega_{0}, \\
\varphi_{n}\left(x_{1}, \pm 0\right)=-\sum_{j=1}^{n} \frac{1}{j !}\left(g_{ \pm}\left(x_{1}\right)\right)^{j} \frac{\partial^{j} \varphi_{n-j}}{\partial x_{2}^{j}}\left(x_{1}, \pm 0\right), \quad x_{1} \in(0,1) .
\end{gathered}
$$

Пусть $j$ - любое полуцелое число, $H_{j}(x)$ - однородные функции порядка $j$ из $C^{\infty}\left(\mathbb{R}^{2} \backslash l_{+}\right)$, где $l_{+}-$полуось $x_{2}=0, x_{1} \geqslant 0$. Множество рядов вида

$$
H(x)=\sum_{j=-m}^{\infty} H_{j / 2}(x)
$$

обозначим через $\widetilde{\mathscr{H}}_{m}$. Слагаемые отрицательной степени однородности будем называть сингулярной частью ряда.

Аналогично, множество рядов вида

$$
h(x)=\sum_{j=-m}^{\infty} \alpha_{j} x_{1}^{j / 2}
$$


обозначим через $\widetilde{\mathscr{A}}_{m}$.

ОПРЕДЕЛЕНИЕ. Числовую последовательность

$$
\left\{b_{j}=\frac{1}{2 \pi r^{j / 2}} \int_{0}^{2 \pi} H_{j / 2}(x) \sin \frac{j \theta}{2} d \theta\right\}_{j=1}^{\infty}
$$

будем называть гармонической серией ряда (6.4).

ЛЕмма 6.1. Пусть ряд $H \in \widetilde{\mathscr{H}}_{0}$ имеет нулевую гармоническую серию и является формальным асимптотическим решением при $r \rightarrow 0$ краевой задачи

$$
\left(\Delta+\lambda_{0}\right) H=0, \quad x \in \mathbb{R}^{2} \backslash l_{+}, \quad H\left(x_{1}, \pm 0\right)=0, \quad x_{1}>0 .
$$

Тогда $H=0$.

ДокАЗАТЕЛЬСтво. Следует учесть, что члены ряда из $\widetilde{\mathscr{H}}_{0}$ имеют вид: $r^{j / 2} \Phi_{j}(\theta), j \geqslant 0$. После подстановки в уравнение получается обыкновенное дифференциальное уравнение для $\Phi_{j}$. Из явного вида решений этих уравнений, краевых условий при $\theta=0, \theta=2 \pi$ и из равенств нулю чисел $b_{j}$ следует утверждение леммы.

СлеДСтвиЕ. Пусть $F \in \widetilde{\mathscr{H}}_{m}, h_{ \pm} \in \widetilde{\mathscr{A}}_{m}$, яяды $H^{(1)}, H^{(2)} \in \widetilde{\mathscr{H}}_{m}$ являются формальньми асимптотическими решениями при $r \rightarrow 0$ краевой задачи

$$
\left(\Delta+\lambda_{0}\right) H=F, \quad x \in \mathbb{R}^{2} \backslash l_{+}, \quad H\left(x_{1}, \pm 0\right)=h_{ \pm}\left(x_{1}\right), \quad x_{1}>0,
$$

имеют совпадающие гармонические серии и $H^{(1)}-H^{(2)} \in \widetilde{\mathscr{H}}_{0}$.

Тогда $H^{(1)}=H^{(2)}$.

Обозначим через $\mathscr{H}_{m}$ подмножество функций из $C^{\infty}\left(\bar{\Omega}_{0} \backslash\left\{t_{+} \cup t_{-}\right\}\right)$, где $t_{ \pm}-$ концы разреза $\omega_{0}$, асимптотики которых в точках $t_{ \pm}$(записанные, соответственно, в системах координат $x_{+}=x$ и $\left.x_{-}=\left(1-x_{1}, x_{2}\right)\right)$ принадлежат классу $\widetilde{\mathscr{H}}_{m}$. Аналогично, через $\mathscr{A}_{m}$ обозначим подмножество функций из $C^{\infty}((0,1))$, имеющих на концах интервала асимптотики из класса $\widetilde{\mathscr{A}}_{m}$.

Рассмотрим краевую задачу

$$
\left(\Delta+\lambda_{0}\right) u=F+\lambda \varphi_{0}, \quad x \in \Omega_{0}, \quad u\left(x_{1}, \pm 0\right)=h_{ \pm}\left(x_{1}\right), \quad x_{1} \in(0,1),
$$

где $F \in \mathscr{H}_{m}, h_{ \pm} \in \mathscr{A}_{m}, m \in \mathbb{N}$.

ЛЕмма 6.2. Пусть для любой пары последовательностей $\left\{b_{j}^{ \pm}\right\}_{j=1}^{\infty}$ и любого числа $\lambda$ существует пара рядов $H_{ \pm}\left(x_{ \pm} ;\left\{a_{j}^{ \pm}\right\}_{j=1}^{\infty}, \lambda\right)$ из класса $\widetilde{\mathscr{H}}_{m}$, обладающих следующими свойствами:

а) $\left\{b_{j}^{ \pm}\right\}_{j=1}^{\infty}-$ гармоническая серия ряда $H_{ \pm}\left(x_{ \pm} ;\left\{b_{j}^{ \pm}\right\}_{j=1}^{\infty}, \lambda\right)$;

б) ряд $H_{ \pm}\left(x_{ \pm} ;\left\{b_{j}^{ \pm}\right\}_{j=1}^{\infty}, \lambda\right)$ является асимптотическим решением задачи (6.5) nри $x_{ \pm} \rightarrow 0$;

в) $H_{ \pm}\left(x_{ \pm} ;\left\{\widetilde{b}_{j}^{ \pm}\right\}_{j=1}^{\infty}, \lambda\right)-H_{ \pm}\left(x_{ \pm} ;\left\{\widehat{b}_{j}^{ \pm}\right\}_{j=1}^{\infty}, \lambda\right) \in \widetilde{\mathscr{H}}_{0}$ для любих последовательностей $\left\{\widetilde{b}_{j}^{ \pm}\right\}_{j=1}^{\infty} u\left\{\widehat{b}_{j}^{ \pm}\right\}_{j=1}^{\infty}$.

Тогда существуют постоянная $\lambda$ и функиия $u \in \mathscr{H}_{m}$ такие, что и является решением краевой задачи (6.5) и при $x_{ \pm} \rightarrow 0$ имеет асимптотики, совпадающие с $H_{ \pm}$при некоторых значениях параметров $b_{j}^{ \pm}$. 
ДокАЗАТЕЛЬСтво. Это утверждение доказывается при помощи рассуждений, аналогичных приведенным при доказательстве леммы 4.1. Решение краевой задачи (6.5) будем искать в виде

$$
u(x)=u_{N}(x)=\chi\left(r_{+}\right) H_{N}^{+}\left(x_{ \pm}\right)+\chi\left(r_{-}\right) H_{N}^{-}\left(x_{ \pm}\right)+U_{N}(x)
$$

где $r_{ \pm}=\left|x_{ \pm}\right|, H_{N}^{ \pm}$- частичные суммы (до степеней $r_{ \pm}^{N}$ включительно) рядов $\widehat{H}_{ \pm}\left(x_{ \pm}\right)=H_{ \pm}\left(x ;\left\{b_{j}^{ \pm}=0\right\}_{j=1}^{\infty}, 0\right)$. Подставляя (6.6) в (6.5), получаем краевую задачу для $u_{N}$

$$
\left(\Delta+\lambda_{0}\right) U_{N}=F_{N}+\lambda \varphi_{0}, \quad x \in \Omega_{0}, \quad U_{N}\left(x_{1}, \pm 0\right)=h_{N}^{ \pm}\left(x_{1}\right), \quad x_{1} \in(0,1) .
$$

Здесь функция $F_{N} \in C^{N-2}\left(\bar{\Omega}_{0}\right)$ (где разрез $\omega_{0}$ понимается как двусторонний) имеет нули порядка $r_{ \pm}^{N-2}$ на концах разреза, а функции $h_{N}^{+}, h_{N}^{-} \in C^{N-1}([0,1])$ имеют нули порядка $x_{1}^{N-1}$ и $\left(1-x_{1}\right)^{N-1}$ на соответствующих концах интервала $(0,1)$. Следовательно, сушествует постоянная $\lambda=\lambda(N)$, при которой краевая задача (6.7) разрешима в классе функций $W_{2}^{1}\left(\Omega_{0}\right)$. С другой стороны, подставляя $u_{N_{1}}-u_{N_{2}} \in W_{2}^{1}\left(\Omega_{0}\right)$ в (6.5), легко видеть, что, во-первых, $\lambda$ не зависит от $N$, а во-вторых, функции $u_{N}$ при разных значениях $N$ равны друг другу с точностью до слагаемого, равного собственной функции. В силу произвола в выборе $N$, результатов работы [14] и леммы 3.1 отсюда следует, что существует решение $u \in \mathscr{H}_{m}$ краевой задачи (6.5) (при некоторой постоянной $\lambda$ ), имеющее на конщах разреза асимптотики вида

$$
u(x)=\widetilde{H}_{ \pm}\left(x_{ \pm}\right), \quad x_{ \pm} \rightarrow 0,
$$

где $\widetilde{H}_{ \pm}$отличается от $\widehat{H}_{ \pm}$на слагаемое из $\mathscr{H}_{0}$.

Пусть $\left\{b_{j}^{ \pm}\right\}$- гармоническая серия ряда $\widetilde{H}_{ \pm}$. В силу следствия леммы 6.1 ряды $\widetilde{H}_{ \pm}\left(x_{ \pm}\right)$и $H_{ \pm}\left(x_{ \pm} ;\left\{b_{j}^{ \pm}\right\}_{j=1}^{\infty}, \lambda\right)$ совпадают. Лемма доказана.

\section{§7. Согласование разложений в случае параболических концов щели}

На формальных рядах $V(\xi ; \varepsilon)$ вида:

$$
V(\xi ; \varepsilon)=\sum_{i \geqslant 0} \sum_{j \geqslant 0} \varepsilon^{i} \ln ^{j} \varepsilon V_{i, j}(\xi)
$$

определим операторы переразложения $\mathscr{M}^{ \pm}$следующим стандартным образом. Коэффициенты ряда $V$ заменяем на их асимптотики на бесконечности и приходим к переменньм $x_{ \pm}=\varepsilon^{2} g_{1}^{ \pm 2} \xi$. Полученный двойной формальный ряд и назовем значением $\mathscr{M}^{ \pm}(V(\xi ; \varepsilon))$.

Обозначим через $v^{ \pm}(\xi ; \varepsilon)$ ряд (5.2), записанный во внутренних переменных левого и правого конца щели, соответственно. Из леммы 5.3 и определения операторов переразложения вытекает

ЛЕмма 7.1. Пусть $\left\{\lambda_{m}\right\}_{m=1}^{\infty}-$ произвольная последовательность действительных чисел и коэффициенты ряда $v^{ \pm}$удовлетворяют утверждениям 
леммы 5.3 (при добавлении в ее формулировке индексов “土”, соответствующих $\left.\xi=x_{ \pm} \varepsilon^{-2}\left(g_{1}^{ \pm}\right)^{-2}\right)$. Тогда справедливо представление

$$
\mathscr{M}^{ \pm}\left(v^{ \pm}(\xi ; \varepsilon)\right)=\sum_{j=0}^{\infty} \varepsilon^{j} \Phi_{j}^{ \pm}(x)
$$

Рядъи $\Phi_{n}^{ \pm} \in \widetilde{\mathscr{H}}_{n-1}$ являются формальными асимптотическими решениями $\left(\right.$ при $\left.x_{ \pm} \rightarrow 0\right)$ рекуррентной системы краевых задач (6.2), (6.3), где в правых частях уравнений и граничных условий функции $\varphi_{j}$ заменень на ряды $\Phi_{j}^{ \pm}$.

Гармоническая серия $\left\{\stackrel{ \pm}{b}_{i}^{(n)}\right\}_{i=1}^{\infty}$ ряда $\Phi_{n}^{ \pm}$имеет вид: ${ }_{b}^{ \pm}{ }_{i}^{(n)}=\stackrel{ \pm}{a}_{i}^{(n+i)} g_{1}^{-i}$.

Ряды $\Phi_{n}^{ \pm}$не зависят от $\stackrel{ \pm}{a}_{i}^{(m+i)}$ и $\lambda_{m}$ nри $m>n$, а их сингулярные части не зависят и от $\stackrel{ \pm}{a}_{i}^{(n+i)}$ u $\lambda_{n}$.

Из утверждений лемм 5.3, 6.2 и 7.1 вытекает

ТЕОРема 7.1. Существуют ряды (5.1), (5.2) и (6.1) и последовательности $\left\{{\stackrel{ \pm}{a_{i}}}_{i}^{(n)}\right\}, 1 \leqslant i \leqslant n$, обладающие следующими свойствами:

а) $\varphi_{n} \in \mathscr{H}_{n-1}$ являются решениями (6.2), (6.3);

б) ряды (5.2) удовлетворяют утвержсдениям леммы $5.3\left(\right.$ при $\left.a_{i}^{(n)}=\stackrel{ \pm}{a}_{i}^{(n)}\right)$;

в) $\mathscr{M}^{ \pm}\left(v^{ \pm}(\xi ; \varepsilon)\right)=\sum_{j=0}^{\infty} \varepsilon^{j} \varphi_{j}^{ \pm}(x)$ npu $x_{ \pm} \rightarrow 0$.

СлЕДСТВИЕ. Коэффиииенты $\lambda_{1}, \lambda_{2} u v_{1}^{ \pm}$определяются равенствами (3.6), (4.14), (3.8) u (4.6).

Коэффичиент $\varphi_{1}$ определен в лемме 3.2 .

Коэффициент $\varphi_{2}$ имеет вид (4.13), где $\psi_{ \pm}$определень в лемме 4.1, а $\widetilde{\varphi}-$ в замечании 3.1 .

Справедливость последнего утверждения следует из построений, приведенных в третьем и четвертом параграфах, и следствия леммы 6.1.

Из теоремы 7.1 и ее следствия вытекает, что в случае параболических концов щели ряды (6.1) и (5.1) являются асимптотическим решением задачи (1.1) при $r_{+}>\varepsilon, r_{-}>\varepsilon$, а ряды (5.2) и (5.1) являются асимптотическим решением задачи (1.1) при $r_{ \pm}<2 \varepsilon$.

\section{§8. Случай общего положения}

В случае общего положения уравнение границы $\partial \omega_{\varepsilon}$ в окрестности концов разреза во внутренних переменных имеет вид:

$$
\xi_{1}=\xi_{2}^{2}+\varepsilon \sum_{i=0} \varepsilon^{i} c_{i+3} \xi_{2}^{i+3}, \quad \varepsilon \xi_{2} \rightarrow 0
$$

где на правом конце $c_{3}=-2 g_{2}^{+}$, на левом конще $c_{3}=-2 g_{2}^{-}$. Сначала попытаемся найти асимптотические разложения собственной функции и собственного значения, по-прежнему, в виде (5.1), (5.2), (6.1) (хотя, как будет показано ниже, правильное разложение этих величин носит более сложный характер). Ясно, что в 
этом случае краевые задачи для коэффициентов ряда (6.1) и уравнения для коэффициентов ряда (5.2) сохранят вид (6.2), (6.3) и (5.4), соответственно. Однако, подставляя (5.2) и (8.1) в граничные условия в (1.1) и переходя к внутренним переменньм, для коэффициентов ряда (5.2) получаем уже неоднородные граничные условия

$$
v_{n}=-\sum_{i=1}^{n-1} \sum_{j=0}^{i-1} c_{n, i, j} \frac{\partial^{i-j} v_{n-i}}{\partial \xi_{1}^{i-j}} \xi_{2}^{3 i-2 j}, \quad \xi \in \partial \Pi,
$$

где постоянные $c_{n, i, j}$ явно определяются через коэффициенты (8.1) или, что то же самое, через коэффициенты (1.2). В частности,

$$
c_{k, 1,0}=2 g_{2}^{ \pm}, \quad c_{k, 2,0}=-2\left(g_{2}^{ \pm}\right)^{2} .
$$

Коэффициенты ряда (5.2) будем вновь строить в виде (5.6). Из (8.2) следует, что однородные граничные условия для $v_{1}$ сохраняются и, следовательно, $v_{1}$, по-прежнему, определяется равенством $v_{1}=a_{1}^{(1)} X_{1}$. Однако, из (8.2) также следует, что граничное условие для второго коэффициента уже неоднородно и имеет вид:

$$
v_{2}=-\frac{1}{2} a_{1}^{(1)} c_{2,1,0} \xi_{2}^{3} \operatorname{Im} z^{-1 / 2}, \quad \xi \in \partial \Pi .
$$

Обозначим $Y_{2}(\xi)=-\frac{1}{2} \operatorname{Re} z^{1 / 2}+\frac{1}{8} \operatorname{Re} z^{-1 / 2}$. Учитывая, что $|z|=\xi_{2}^{2}+\frac{1}{4}$ на $\partial П$, легко видеть, что функция

$$
\widetilde{v}_{2}=-\frac{1}{2} a_{1}^{(1)} c_{2,1,0} Y_{2}
$$

(а следовательно, и функция $v_{2}$ ) является решением (5.4), (8.2). При исследовании разрешимости краевых задач с подобными граничными условиями будет использоваться следующий, легко проверяемый, аналог леммы 5.2.

ЛЕмма 8.1. Для любых целых положсительных $k$ и т краевые задачи

$$
\begin{aligned}
& \Delta v=0, \quad \xi \in \Pi, \quad v=\xi_{2}^{k} \operatorname{Im} z^{-m / 2}, \quad \xi \in \partial \Pi, \\
& \Delta w=0, \quad \xi \in \Pi, \quad w=\xi_{2}^{k} \operatorname{Re} z^{-m / 2}, \quad \xi \in \partial \Pi,
\end{aligned}
$$

имеют решения вида

$$
\begin{aligned}
v(\xi) & =\sum_{j=0}^{[(k-1) / 2]} \alpha_{k, m, j} \operatorname{Re} z^{\frac{k-m-1}{2}-j}+\sum_{j=\max \{0 ;[(k-m) / 2]+1\}}^{[k / 2]} \sum_{k, m, j} \operatorname{Im} z^{\frac{k-m}{2}-j}, \\
w(\xi) & =\sum_{j=0}^{[k / 2]} \gamma_{k, m, j} \operatorname{Re} z^{\frac{k-m}{2}-j}+\sum_{j=\max \{0 ;[(k-1) / 2]}^{[(k-m+1) / 2]\}} \delta_{k, m, j} \operatorname{Im} z^{\frac{k-m-1}{2}-j},
\end{aligned}
$$

əде $\alpha_{3,1,1}=\frac{1}{8}, \gamma_{3,3,1}=-\frac{3}{4}, \alpha_{6,3,2}=\frac{5}{16}$.

Из (8.2) и леммы 8.1 (см. также (8.4)), в частности, следует, что

$$
\begin{gathered}
\widetilde{v}_{3}(\xi)=b_{2} \operatorname{Re} z+b_{1} \operatorname{Re} z^{1 / 2}+b_{0}+b_{-1} \operatorname{Re} z^{1 / 2}+a_{1}^{(1)} A \operatorname{Re} z^{-1} \\
+d_{-1} \operatorname{Im} z^{-1 / 2}+d_{-3} \operatorname{Im} z^{-3 / 2} \\
A=\frac{1}{128}\left(5 c_{3,2,0}+3 c_{3,1,0} c_{2,1,0}\right)
\end{gathered}
$$


Из определения $v_{1}$ и $v_{2}$ (см. (8.4)) и утверждений лемм 5.1, 5.2 и 8.1 следует, что при $n<7$ краевые задачи $(5.4),(8.2)$ для $\widetilde{v}_{n}$ разрешимы в классе степенных функций. Однако при $n=7$ правая часть в (5.4) в силу (8.5) содержит резонансный член вида:

$$
-\Delta v_{7}=\cdots+\lambda_{0} A a_{1}^{(1)} \operatorname{Re} z^{-1}+\cdots,
$$

где многоточием обозначены члены, содержащие степени $z$, отличные от -1 .

Следующие два утверждения являются легко проверяемыми аналогами лемм 5.1 и 5.2 .

Лемма 8.2. Для любъх $k$ u $m$, отличных от -1, и любъх иельх $n \geqslant 1$ уравнение $\Delta v=\bar{z}^{k} z^{m} \ln ^{n} z$ имеет решение вида

$$
v(\xi)=\bar{z}^{k+1} z^{m+1} \sum_{j=0}^{n} \alpha_{j} \ln ^{j} z
$$

Для любого челого $n \geqslant 0$ уравнение $\Delta v=z^{-1} \ln ^{n} z$ имеет решение вида

$$
v(\xi)=\frac{1}{4(n+1)} \bar{z} \ln ^{n+1} z .
$$

ЛЕмма 8.3. Для любъх иельх положсительных $k$ и $n$ краевые задачи

$$
\begin{aligned}
& \Delta v=0, \quad \xi \in \Pi, \quad v=\xi_{2}^{k} \operatorname{Re} \ln ^{n} z, \quad \xi \in \partial \Pi, \\
& \Delta w=0, \quad \xi \in \Pi, \quad w=\xi_{2}^{k} \operatorname{Im} \ln ^{n} z \quad \xi \in \partial \Pi,
\end{aligned}
$$

имеют решения вида

$$
\begin{aligned}
v(\xi) & =\sum_{j=0}^{[k / 2]} \alpha_{k, j} \operatorname{Re}\left(z^{\frac{k}{2}-j} \ln ^{n} z\right)+\sum_{j=0}^{[(k-1) / 2]} \beta_{k, j} \operatorname{Im}\left(z^{\frac{k-1}{2}-j} \ln ^{n} z\right), \\
w(\xi) & =\sum_{j=0}^{[k / 2]} \gamma_{k, j} \operatorname{Im}\left(z^{\frac{k}{2}-j} \ln ^{n} z\right)+\sum_{j=0}^{[(k-1) / 2]} \delta_{k, j} \operatorname{Re}\left(z^{\frac{k-1}{2}-j} \ln ^{n} z\right) .
\end{aligned}
$$

Непосредственно проверяется

СлЕДСТВИЕ. Для любого иелого $n \geqslant 0$ функиия

$$
v(\xi)=\frac{1}{4(n+1)}\left(\operatorname{Re}\left((\bar{z}-z) \ln ^{n+1} z\right)-2 \operatorname{Im}\left(z^{1 / 2} \ln ^{n+1} z\right)+\operatorname{Re} \ln ^{n+1} z\right)
$$

или, что то жее самое,

$$
v(\xi)=A_{n+1}\left(X_{1}(\xi) \operatorname{Re} \ln ^{n+1} z+\left(\operatorname{Re} z^{1 / 2}-\xi_{2}\right) \operatorname{Im} \ln ^{n+1} z\right), \quad A_{m}=-\frac{1}{2 m}
$$

является решением краевой задачи

$$
\Delta v=\operatorname{Re}\left(z^{-1} \ln ^{n} z\right), \quad \xi \in \Pi, \quad v=0, \quad \xi \in \partial \Pi
$$


Из (8.6) и леммы 8.2 следует, что первая степень логарифма появляется при решении (5.4), (8.2) для $v_{7}$. Из следствия леммы 8.3 вытекает, что логарифмические слагаемые в представлении функции $\widetilde{v}_{7}$ имеют вид:

$$
\begin{gathered}
\widetilde{v}_{7}(\xi)=\cdots+\widetilde{a}_{7}\left(\operatorname{Im}\left(z^{1 / 2} \ln z\right)-\frac{1}{2} \operatorname{Re} \ln z\right)-\frac{1}{2}(\operatorname{Re}((\bar{z}-z) \ln z))+\cdots, \\
\widetilde{a}_{7}=-\lambda_{0} A_{1} A a_{1}^{(1)}
\end{gathered}
$$

или (в другой форме записи)

$$
\widetilde{v}_{7}(\xi)=\cdots+\widetilde{a}_{7} X_{1}(\xi) \operatorname{Re} \ln z+\alpha\left(\operatorname{Re} z^{1 / 2}-\xi_{2}\right) \operatorname{Im} \ln z+\cdots,
$$

где многоточием обозначены степенные члены.

Непосредственно проверяется следующий аналог лемм 8.1 и 8.3.

ЛЕмма 8.4. Для любых целых положительных $k, m$ и п краевые задачи

$$
\begin{aligned}
& \Delta v=0, \quad \xi \in \Pi, \quad v=\xi_{2}^{k} \operatorname{Im}\left(z^{-m / 2} \ln ^{n} z\right), \quad \xi \in \partial \Pi, \\
& \Delta w=0, \quad \xi \in \Pi, \quad w=\xi_{2}^{k} \operatorname{Re}\left(z^{-m / 2} \ln ^{n} z\right), \quad \xi \in \partial \Pi,
\end{aligned}
$$

имеют решения вида

$$
\begin{aligned}
v(\xi) & =\sum_{j=0}^{[(k-1) / 2]} \alpha_{k, m, j} \operatorname{Re}\left(z^{\frac{k-m-1}{2}-j} \ln ^{n} z\right)+\sum_{j=0}^{[k / 2]} \beta_{k, m, j} \operatorname{Im}\left(z^{\frac{k-m}{2}-j} \ln ^{n} z\right), \\
w(\xi) & =\sum_{j=0}^{[k / 2]} \gamma_{k, m, j} \operatorname{Re}\left(z^{\frac{k-m}{2}-j} \ln ^{n} z\right)+\sum_{j=0}^{[(k-1) / 2]} \delta_{k, m, j} \operatorname{Im}\left(z^{\frac{k-m}{2}-j} \ln ^{n} z\right),
\end{aligned}
$$

где постоянные $\alpha_{3,1,1}, \gamma_{3,3,1}, \alpha_{6,3,2}$ имеют те жее значения, что и в лемме 8.1.

С учетом леммы 8.4 легко видеть, что для дальнейшего увеличения степеней логарифма первое слагаемое в (8.7) играет ту же роль, что и слагаемое $a_{1}^{(1)} \operatorname{Im} z^{1 / 2}$ (в определении $v_{1}$ ) для появления логарифма. Используя утверждения лемм 8.2-8.4, получаем следующую цепочку, отслеживающую нарастание 
степеней логарифма у коэффициентов ряда (5.2):

$$
\begin{aligned}
(8.7) \Rightarrow & \widetilde{v}_{9}(\xi)=\cdots+\widetilde{a}_{7} A \operatorname{Re}\left(z^{-1} \ln z\right)+\cdots \\
\Rightarrow & -\Delta v_{13}=\cdots+\lambda_{0} \widetilde{a}_{7} A \operatorname{Re}\left(z^{-1} \ln z\right)+\cdots \\
\Rightarrow & \widetilde{v}_{13}(\xi)=\cdots+\widetilde{a}_{13}\left(\operatorname{Im}\left(z^{1 / 2} \ln ^{2} z\right)-\frac{1}{2} \operatorname{Re}^{2} \ln ^{2}\right. \\
& \left.\quad-\frac{1}{2}\left(\operatorname{Re}\left((\bar{z}-z) \ln ^{2} z\right)\right)\right)+\cdots, \\
\Rightarrow & \widetilde{v}_{1+6 n}(\xi)=\cdots+\widetilde{a}_{1+6 n}\left(\operatorname{Im}\left(z^{1 / 2} \ln ^{n} z\right)-\frac{1}{2} \operatorname{Re} \ln ^{n} z\right. \\
& \left.\widetilde{a}_{1+6 n}=-\frac{1}{2} \operatorname{Re}\left((\bar{z}-z) \ln ^{n} z\right)\right)+\cdots, \\
\Rightarrow & \widetilde{v}_{3+6 n}(\xi)=\cdots+\widetilde{a}_{1+6 n} A \operatorname{Re}\left(z^{-1} \ln ^{n} z\right)+\cdots \\
\Rightarrow & -\Delta v_{1+6(n+1)}=\cdots+\lambda_{0} \widetilde{a}_{1+6 n} A \operatorname{Re}\left(z^{-1} \ln ^{n} z\right)+\cdots \\
\Rightarrow & \widetilde{v}_{1+6(n+1)}(\xi)=\cdots+\widetilde{a}_{1+6(n+1)}\left(\operatorname{Im}\left(z^{1 / 2} \ln ^{n+1} z\right)-\frac{1}{2} \operatorname{Re} \ln ^{n+1} z\right. \\
& \left.\quad-\frac{1}{2} \operatorname{Re}\left((\bar{z}-z) \ln ^{n+1} z\right)\right)+\cdots, \\
& \widetilde{a}_{1+6(n+1)}=-\lambda_{0} A A_{n+1} \widetilde{a}_{1+6 n},
\end{aligned}
$$

или в другой форме записи

$$
\begin{aligned}
\widetilde{v}_{1+6(n+1)}(\xi)=\cdots+\widetilde{a}_{1+(6 n+1)} X_{1}(\xi) & \operatorname{Re} \ln ^{n+1} z \\
& +\alpha\left(\operatorname{Re} z^{1 / 2}-\xi_{2}\right) \operatorname{Im} \ln ^{n+1} z+\cdots
\end{aligned}
$$

где многоточие обозначает слагаемые с меньшими степенями логарифма.

Из (8.9) и (8.7) следует, что

$$
\widetilde{a}_{1+6 m}=\left(-\lambda_{0} A\right)^{m} \prod_{j=1}^{m} A_{j} a_{1}^{(1)} .
$$

Для рядов (5.1), (5.2) сформулируем аналог леммы 5.3, вытекающий из утверждений лемм 5.1, 5.2, 8.1-8.4.

ЛЕмма 8.5. Пусть выполнень условия леммы 5.3. Тогда система краевых задач (5.4), (8.2) имеет систему решений, которые могут бъть представле- 
нъь в виде (5.6), әде

$$
\begin{aligned}
\widetilde{v}_{n}(\xi)=\sum_{j=1}^{n} \beta_{n, 0, k, j} \operatorname{Re} z^{j / 2} & \\
& +\sum_{k=1}^{[(n-1) / 4]} \sum_{j=1}^{n-4 k}|z|^{2 k}\left(\alpha_{n, 0, k, j} \operatorname{Im} z^{j / 2}+\beta_{n, 0, k, j} \operatorname{Re} z^{(j-1) / 2}\right) \\
& +\sum_{k=0}^{[(n-1) / 4]} \sum_{j=1}^{n-3-8 k}|z|^{2 k}\left(\alpha_{n, 0, k,-j} \operatorname{Im} z^{-j / 2}+\beta_{n, 0, k,-j} \operatorname{Re} z^{-j / 2}\right) \\
& +\sum_{m=1}^{[(n-1) / 6][(n-6 m-4 k+3) / 4] n-6 m-4 k-1} \mid \sum_{k=0}^{j=\min \{0 ; 3-2(n-6 m-4 k)\}}\left(\alpha_{n, m, k, j} \operatorname{Im}\left(z^{j / 2} \ln ^{m} z\right)\right. \\
& \left.+\sum_{m=1}^{[(n-1) / 6][(n-6 m-4 k+3) / 4]} \sum_{n, m, k, j} \operatorname{Re}\left(z^{j / 2} \ln ^{m} z\right)\right)
\end{aligned}
$$

а постоянные $\alpha_{n, i, k, j}, \beta_{n, i, k, j}, \gamma_{n, i, k}$ и $\delta_{n, i, k}$ не зависят от $a_{s}^{(m)}$ nри $m \geqslant n$.

Для $\widetilde{v}_{1+6 m}$ справедливы представления (8.10), (8.11), где опущены слагаемъе с меньиими степенями логарифма.

Перейдем к внешнему разложению. Множество рядов вида

$$
H(x)=\sum_{j=-m}^{\infty} H_{j / 2}(x)+\sum_{j=0}^{\infty} \sum_{i=1}^{n_{j}} \ln ^{i} r P_{j / 2}^{(i)}(x),
$$

где $P_{j}(x)$ - однородные функции порядка $j$ из $C^{\infty}\left(\mathbb{R}^{2} \backslash l_{+}\right), n_{j}$ - произвольные натуральные числа, будем, по-прежнему, обозначать через $\widetilde{\mathscr{H}}_{m}$. Аналогично, для множества рядов вида

$$
h(x)=\sum_{j=-m}^{\infty} \alpha_{j} x_{1}^{j / 2}+\sum_{j=0}^{\infty} \sum_{i=1}^{n_{j}} \ln ^{i} x_{1} \beta_{j, i} x_{1}^{j}
$$

оставим прежнее обозначение $\tilde{\mathscr{A}}_{m}$. Определения сингулярной части и гармонической серии ряда (8.13) - те же, что и для ряда (6.4). Определения $\mathscr{H}_{m}$ и $\mathscr{A}_{m}$ сохранены. Из новых определений множеств $\widetilde{\mathscr{H}}_{m}$ и $\widetilde{\mathscr{A}}_{m}$ вытекает

Лемма 8.6. В новых определениях множсеств $\widetilde{\mathscr{H}}_{m}$ и $\widetilde{\mathscr{A}}_{m}$ остаются справедливыми все утверждения леммы 6.1, ее следствия и леммы 6.2.

Прежде чем перейти к согласованию разложений, на суммах $V(\xi ; \varepsilon)$ вида

$$
V(\xi ; \varepsilon)=\sum_{i \geqslant 0} \sum_{j \geqslant 0} \varepsilon^{i} \ln ^{j} \varepsilon V_{i, j}(\xi)
$$

определим оператор $\mathscr{M}_{m}^{ \pm}$следующим образом: оставим в ряду $\mathscr{M}^{ \pm}(V(\xi ; \varepsilon))$ только члены вида $\varepsilon^{i} \ln \varepsilon^{j} \Phi(x)$ при $j \leqslant m$. Этот формальный ряд определим как $\mathscr{M}_{m}^{ \pm}(V(\xi ; \varepsilon))$. Из леммы 8.5 и определения операторов переразложения вытекает аналог леммы 7.1. 
ЛЕМма 8.7. Пусть $\left\{\lambda_{m}\right\}_{m=1}^{\infty}-$ произвольная последовательность действительных чисел и ряд $v^{ \pm}$удовлетворяет утверждениям леммь 8.5 ( с добавлением в ее формулировке индексов “土”, соответствующих $\left.\xi=x_{ \pm} \varepsilon^{-2}\left(g_{1}^{ \pm}\right)^{-2}\right)$. Тогда справедливы представление

$$
\mathscr{M}_{0}^{ \pm}\left(v^{ \pm}(\xi ; \varepsilon)\right)=\sum_{j=0}^{\infty} \varepsilon^{j} \Phi_{j}^{ \pm}(x)
$$

(вместо представления (7.1)) и все остальные утверждения леммы 7.1 .

Подчеркнем, что из (8.8) вытекает равенство

$$
\mathscr{M}_{1}^{ \pm}\left(v^{ \pm}(\xi ; \varepsilon)\right)=\mathscr{M}_{0}^{ \pm}\left(v^{ \pm}(\xi ; \varepsilon)\right)-2 \varepsilon^{7} \ln \varepsilon\left(g_{1}^{ \pm}\right)^{-1} \stackrel{\stackrel{a}{a}_{7}}{r_{ \pm}^{1 / 2}} \sin \frac{\theta_{ \pm}}{2}+O\left(\varepsilon^{8} \ln \varepsilon\right)
$$

и следовательно, из равенства (8.14) для построенных рядов не следует равенство (7.1), соответствуюшее полностью согласованным разложениям. Для устранения возникшей в (8.15) невязки приходится вводить во внутреннем разложении члены $\varepsilon^{7} \ln \varepsilon v_{7,1}^{ \pm}$, которые, в свою очередь, приводят к появлению степеней $\ln \varepsilon$ во внешнем разложении и, следовательно (в силу условия разрешимости соответствуюших краевых задач внешнего разложения), к появлению степеней $\ln \varepsilon$ и в асимптотике собственного значения. По этим причинам полные асимптотики собственного значения и собственной функции будем искать в виде:

$$
\begin{gathered}
\lambda_{\varepsilon}=\lambda_{0}+\varepsilon \lambda_{1}+\sum_{i=2}^{\infty} \sum_{j=0}^{[(i-2) / 6]} \varepsilon^{i} \ln ^{j} \varepsilon \lambda_{i, j}, \\
\varphi_{\varepsilon}(x)=\varphi_{0}(x)+\varepsilon \varphi_{1}(x)+\sum_{i=2}^{\infty} \sum_{j=0}^{[(i-2) / 6]} \varepsilon^{i} \ln ^{j} \varepsilon \varphi_{i, j}(x), \quad x \in \Omega_{0} \backslash S(\varepsilon), \\
\varphi_{\varepsilon}(x)=\sum_{i=1}^{\infty} \sum_{j=0}^{[(i-1) / 6]} \varepsilon^{i} \ln ^{j} \varepsilon v_{i, j}^{ \pm}\left(x_{ \pm}\left(g_{1}^{ \pm} \varepsilon\right)^{-2}\right), \quad x \in S_{ \pm}(2 \varepsilon),
\end{gathered}
$$

где $S(t)=S_{-}(t) \cup S_{+}(t)$. (Напомним, что $S_{ \pm}(t)-$ круг радиуса $t$ с центром в соответствуюшем конце щели.)

Подставляя (8.16) и (8.17) в (1.1), получаем краевые задачи для коэффициентов внешнего разложения:

$$
\begin{gathered}
-\Delta \varphi_{n, m}=\sum_{j, i \geqslant 0}^{n} \lambda_{j, i} \varphi_{n-j, m-i}, \quad x \in \Omega_{0}, \\
\varphi_{n, m}\left(x_{1}, \pm 0\right)=-\sum_{j \geqslant 1} \frac{1}{j !}\left(g_{ \pm}\left(x_{1}\right)\right)^{j} \frac{\partial^{j} \varphi_{n-j, m}}{\partial x_{2}^{j}}\left(x_{1}, \pm 0\right), \quad x_{1} \in(0,1),
\end{gathered}
$$

где $\lambda_{0,0}=\lambda_{0}, \varphi_{0,0}=\varphi_{0}, \lambda_{1,0}=\lambda_{1}, \varphi_{1,0}=\varphi_{1}$. 
Подставляя (8.16) и (8.18) в (1.1) и переходя к внутренним переменным, получаем краевые задачи для коэффициентов внутреннего разложения:

$$
\begin{gathered}
-\Delta v_{n, m}^{ \pm}=\sum_{i, j \geqslant 0} \lambda_{i, j} v_{n-i-4, m-j}, \quad \xi \in \Pi, \\
v_{n, m}^{ \pm}=-\sum_{i=1}^{n-6 m-1} \sum_{j=0}^{i-1} c_{n, i, j} \frac{\partial^{i-j} v_{n-i, m}^{ \pm}}{\partial \xi_{1}^{i-j}} \xi_{2}^{3 i-2 j}, \quad \xi \in \partial \Pi .
\end{gathered}
$$

Полньй аналог леммы 8.5 для рассматриваемого случая, являюшийся также следствием лемм 5.1, 5.2 и 8.1-8.4, имеет следующий вид.

ЛЕмма 8.8. Пусть $0 \leqslant l<\infty, 6 l+1 \leqslant n<\infty,\left\{a_{i}^{(n, l)}\right\}_{i=1}^{n-6 l}-$ семейство произвольных последовательностей действительных чисел. Тогда система краевых задач (8.20) имеет систему решений, которые могут быть представлены в виде

$$
v_{n, l}(\xi)=\sum_{i=1}^{n-6 l} a_{i}^{(n, l)} X_{i}(\xi)+\widetilde{v}_{n, l}(\xi),
$$

где функиии $\widetilde{v}_{n+6 l, l}$ представимы в виде (8.12) с коэффициентами, зависящими от $l$, но не зависящими от $a_{m}^{(s, t)}$ nри $m \geqslant n+6 l$.

Справедливы представления:

$$
\begin{gathered}
\widetilde{v}_{1+6(m+l), l}(\xi)=\cdots+\widetilde{A}_{1+6(m+l), l} X_{1}(\xi) \operatorname{Re} \ln ^{m} z \\
+2 \alpha(m, l)\left(\xi_{2}-\operatorname{Re} z^{1 / 2}\right) \operatorname{Im} \ln ^{m} z+\cdots, \\
\widetilde{A}_{1+6(m+l), l}=\left(-\lambda_{0} A\right)^{m} a_{1}^{(1+6 l, l)} \prod_{j=1}^{m} A_{j}
\end{gathered}
$$

где в (8.21) опущены слагаемые с меньшими степенями логарифма.

В свою очередь, из лемм 4.1, 8.6-8.8 и соотношений $(8.3),(8.5),(8.21)$ и (8.22) вытекает аналог теоремы 7.1 для обшего случая.

ТЕОРема 8.1. Существуют ряды (8.16)-(8.18) и семейство последовательностей $0 \leqslant l<\infty, 6 l+1 \leqslant n<\infty,\left\{\frac{ \pm}{a}_{i}^{(n, l)}\right\}_{i=1}^{n-6 l}$, обладающие следующими свойствами.

Справедливо равенство

$$
\stackrel{ \pm}{a}_{1}^{(6 m+1, m)}=-\sum_{j=0}^{m-1}(-2)^{m-j} \widetilde{A}_{6 m+1, j}^{ \pm}
$$

ฉдe

$$
\widetilde{A}_{6 m+1, j}^{ \pm}=\frac{1}{(m-j) !}\left(-\frac{\lambda_{0}\left(g_{2}^{ \pm}\right)^{2}}{128}\right)^{m-j} \stackrel{ \pm}{a}(6 j+1, j), \quad \stackrel{ \pm}{a}(1,0)=d_{ \pm} g_{1}^{ \pm} .
$$

Функиии $\varphi_{n, m} \in \mathscr{H}_{n-1-6 m}$ являются решениями (8.19). 
Ряды (8.18) удовлетворяют утверждениям леммы $8.8\left(\right.$ при $\left.a_{i}^{(n, l)}=\stackrel{ \pm}{a}_{i}^{(n, l)}\right)$,

$$
\mathscr{M}^{ \pm}\left(v^{ \pm}(\xi ; \varepsilon)\right)=\varphi_{0}(x)+\varepsilon \varphi_{1}(x)+\sum_{i=2}^{\infty} \sum_{j=0}^{[(i-2) / 6]} \varepsilon^{i} \ln ^{j} \varepsilon \varphi_{i, j}(x), \quad x_{ \pm} \rightarrow 0,
$$

əде $v^{ \pm}-$ряды (8.18).

Коэффициенты $\lambda_{1,0}=\lambda_{1}, \lambda_{2,0}=\lambda_{2}, v_{1,0}^{ \pm}=v_{1}^{ \pm}, \varphi_{1,0}=\varphi_{1} u \varphi_{2,0}=\varphi_{2}$ удовлетворяют утверждению следствия теоремы 7.1.

Коэффициенты ряда (8.16), содержащие $m$-ю степень $\ln \varepsilon$ при наименьшей степени є, имеют вид:

$$
\lambda_{6 m+2, m}=\frac{\pi}{8}\left(d_{+} g_{1}^{+} \stackrel{+}{a}_{1}^{(6 m+1, m)}+d_{-} g_{1}^{-} \bar{a}_{1}^{(6 m+1, m)}\right), \quad m>1 .
$$

Из равенств (8.22)-(8.25) получаем явную формулу для членов ряда (8.16), отражаюших нарастание степеней логарифма:

$$
\begin{gathered}
\lambda_{6 m+2, m}=\frac{\pi}{8}\left(-\frac{\lambda_{0}}{64}\right)^{m} c(m)\left(\left(g_{2}^{+}\right)^{2 m}\left(d_{+} g_{1}^{+}\right)^{2}+\left(g_{2}^{-}\right)^{2 m}\left(d_{-} g_{1}^{-}\right)^{2}\right), \\
c(m)=-\sum_{j=0}^{m-1} \frac{1}{(m-j) !} c(j), \quad c(0)=1 .
\end{gathered}
$$

В свою очередь, из (8.26) вытекает равенство

$$
\lambda_{6 m+2, m}=\frac{\pi}{8}\left(\frac{\lambda_{0}}{64}\right)^{m}\left(\left(g_{2}^{+}\right)^{2 m}\left(d_{+} g_{1}^{+}\right)^{2}+\left(g_{2}^{-}\right)^{2 m}\left(d_{-} g_{1}^{-}\right)^{2}\right), \quad m>1 .
$$

\section{§9. Обоснование асимптотик}

Обозначим через $\lambda_{\varepsilon, N}, \varphi_{\varepsilon, N}(x)$ и $v_{\varepsilon, N}^{ \pm}\left(x_{ \pm}\left(\varepsilon g_{1}^{ \pm}\right)^{-2}\right)$ частичные суммы рядов $(5.1),(6.1)$ и (5.2) в случае параболических концов щели и частичные суммы рядов (8.16)-(8.18) в случае обшего положения. Далее обозначим

$$
\begin{aligned}
\Phi_{\varepsilon, N}(x)=\left(1-\chi\left(r_{+} \varepsilon^{-1}\right)\right)\left(1-\chi\left(r_{-} \varepsilon^{-1}\right)\right) \varphi_{\varepsilon, N}(x) \\
\\
+\chi\left(r_{+} \varepsilon^{-1}\right) v_{\varepsilon, N}^{+}\left(x_{+}\left(\varepsilon g_{1}^{+}\right)^{-2}\right)+\chi\left(r_{-} \varepsilon^{-1}\right) v_{\varepsilon, N}^{-}\left(x_{-}\left(\varepsilon g_{1}^{-}\right)^{-2}\right) .
\end{aligned}
$$

Из утверждений теорем 7.1 и 8.1 стандартньм образом (см., например, [9]) следует

Лемма 9.1. Пусть рядъи (5.1), (6.1) и (5.2) удовлетворяют утвержсдениям теоремы 7.1, а ряды (8.16)-(8.18) удовлетворяют утверждениям теоремы 8.1. Тогда функиия $\Phi_{\varepsilon, N}(x)$ является решением краевой задачи

$$
\begin{gathered}
-\Delta \Phi_{\varepsilon, N}=\lambda_{\varepsilon, N} \Phi_{\varepsilon, N}+f_{\varepsilon, N}, \quad x \in \Omega_{\varepsilon}, \\
\Phi_{\varepsilon, N}=0, \quad x \in \partial \Omega, \quad \Phi_{\varepsilon, N}=h_{\varepsilon, N}, \quad x \in \partial \omega_{\varepsilon},
\end{gathered}
$$

где $\left\|f_{\varepsilon, N}\right\|_{\Omega} \leqslant C_{N} \varepsilon^{M}, h_{\varepsilon, N}=O\left(\varepsilon^{M}\right)$ в норме $C^{1}\left(\partial \omega_{\varepsilon}\right)$, а M растет неограниченно вместе $c N$. 
ТЕОРема 9.1. Если концы щели имеют вид парабол, то асимптотика собственного значения имеет вид (5.1), а собственная функция имеет асимптотику (6.1) в $\Omega_{\varepsilon} \backslash S(\varepsilon)$ и асимптотики $(5.2)$ в $S_{ \pm}(2 \varepsilon)$ в норме $W_{2}^{1}\left(\Omega_{\varepsilon}\right)$. Для коэффичиентов рядов (5.1), (5.2) и (6.1) справедливы утверждения теоремы 7.1 и ее следствия.

В случае общего положения асимптотика собственного значения имеет вид (8.16), а собственная функция имеет асимптотику (8.17) в $\Omega_{\varepsilon} \backslash S(\varepsilon)$ и асимптотики $(8.18)$ в $S_{ \pm}(2 \varepsilon)$ в норме $W_{2}^{1}\left(\Omega_{\varepsilon}\right)$. Для коэффициентов рядов (8.16)-(8.18) справедливы утверждения теоремы 8.1 и равенства (8.27).

ДокАЗАТЕльСтво. Обозначим через $\widetilde{\Phi}_{\varepsilon, N}$ - гармоническую в $\Omega_{\varepsilon}$ функцию, равную нулю на $\partial \Omega$ и равную $h_{\varepsilon, N}$ на $\partial \omega_{\varepsilon}$. В силу леммы 9.1 и априорных оценок гармонической функции и ее производных получаем, что

$$
\left\|\widetilde{\Phi}_{\varepsilon, N}\right\|_{1, \Omega_{\varepsilon}}=C_{N} \varepsilon^{M} .
$$

Положим $\widehat{\Phi}_{\varepsilon, N}=\Phi_{\varepsilon, N}-\widetilde{\Phi}_{\varepsilon, N}$. В силу леммы 9.1 и соотношения (9.1) функция $\widehat{\Phi}_{\varepsilon, N}$ является решением краевой задачи

$$
-\Delta \widehat{\Phi}_{\varepsilon, N}=\lambda_{\varepsilon, N} \widehat{\Phi}_{\varepsilon, N}+F_{\varepsilon, N}, \quad x \in \Omega_{\varepsilon}, \quad \Phi_{\varepsilon, N}=0, \quad x \in \partial \Omega_{\varepsilon},
$$

где

$$
\left\|F_{\varepsilon, N}\right\|_{\Omega} \leqslant C_{N} \varepsilon^{M} .
$$

С другой стороны, из построения и оценки (9.1) следует, что

$$
\left\|\widehat{\Phi}_{\varepsilon, N}\right\|_{\Omega} \rightarrow 1
$$

В силу произвольности выбора $N$ из соотношений $(9.1),(9.3)$ и $(9.4)$ и утверждения леммы 2.3 следует справедливость всех утверждений доказываемой теоремы.

\section{Список литературы}

1. Самарский $A . A$. О влиянии закрепления на собственные частоты замкнутых объемов // Докл. АН СССР. 1948. Т. 63. С. 631-634.

2. Ozawa $S$. Singular variation of domains and eigenvalues of the Laplacian // Duke Math. J. 1981. V. 48. P. $767-778$.

3. Мазья В.Г., Назаров С. А., Пламеневский Б. А. Асимптотические разложения собственных чисел краевых задач для оператора Лапласа в областях с мальми отверстиями // Изв. АН СССР. Сер. матем. 1984. Т. 48. № 2. С. 347-371.

4. Гадыльшин P. Р. Асимптотика собственного значения сингулярно возмущенной эллиптической задачи с малым параметром в граничном условии // Дифференц. уравнения. 1986. T. 22. № 4. C. $640-652$.

5. Назаров С. А. Асимптотические разложения собственных чисел. Л.: Изд-во ЛГУ, 1987.

6. Гадыльшин Р. Р. Расщепление кратного собственного значения задачи Дирихле для оператора Лапласа при сингулярном возмущении граничного условия // Матем. заметки. 1992. Т. 52. № 4. С. 42-55.

7. Jimbo S., Morita Y. Remarks on the behavior of certain eigenvalues in the singularly perturbed domain with several thin channels // Comm. Partial Differential Equations. 1992. V. 17. P. 523-552. 
8. Жиков В.В., Козлов С. М., Олейник О.А. Усреднение дифференциальных операторов. М.: Физматлит, 1993.

9. Ильин A. М. Краевая задача для эллиптического уравнения второго порядка в области с узкой щелью. 1. Двумерньй случай // Матем. сб. 1976. Т. 99. № 4. С. 514-537.

10. Ильин A. М. Согласование асимптотических разложений решений краевых задач. М.: Наука, 1989.

11. Ван-Дайк М. Методы возмущений в механике жидкости. М.: Мир, 1967.

12. Найфе A. Х. Методы возмущений. М.: Мир, 1986.

13. Лады женская О. А. Краевые задачи математической физики. М.: Наука, 1973.

14. Кондратьев B. A. Краевые задачи для эллиптических уравнений в областях с коническими и угловьми точками // Труды ММО. 1967. Т. 16. С. 209-292.

Институт математики с ВЦ Уфимского научного центра РАН

Институт математики и механики УрО РАН

Поступила в редакцию

E-mail: grr@imat.rb.ru, iam@eqmph.imm.intec.ru 